\title{
Detection of Human Herpes Virus Type 6 and Varicella Zoster Virus in the Urine of Patients with Multiple Sclerosis in Kerman Province, Iran
}

\author{
Kimia Esmaili, Kumarss Amini* \\ Department of Microbiology, School of Basic Sciences, Saveh Branch, Islamic Azad University, Saveh, Iran
}

Article Info:

Received: 6 Jan 2017

Revised: 13 Jan 2017

Accepted: 3 Jul 2017

\section{ABSTRACT}

Introduction: Multiple sclerosis (MS) is a demyelinating disease of the CNS in which autoimmunity to myelin plays a role in pathogenesis. Infection with common viruses, particularly varicella zoster virus (VZV) and Human herpesvirus 6 (HHV-6) are postulated to contribute to the pathobiology of MS. The aim of this study was to detect of HHV-6 and VZV in the urine samples of patients with MS in the Kerman province, Iran. Materials and Methods: In this cross-sectional study, a total of 60 non-repetitive urine samples from patients with MS and 60 urine samples from healthy people without any neurological disease and urinary tract infection were collected. The infection with HHV- 6 and VZV were inves tigated using multiplex PCR method. Results: Of the 60 urine samples obtained from MS patients, $23(13.8 \%)$ and 7 (4.2\%) were positive for HHV-6 and VZV, respectively. VZV and HHV-6 were not detected simultaneously in any of obtained samples. HHV-6 and VZV were not found in any samples obtained from healthy people. Conclusion: The prevalence of HHV-6 and VZV viruses in MS patients is high, which may suggest an association between MS and the presence of these viruses in this region.

Key words:

1. Multiple Sclerosis

2. Herpesvirus 6, Human

3. Multiplex Polymerase

Chain Reaction

4. Autoimmunity

*Corresponding Author: Kumarss Amini

E-mail: $d r \_k u m a r s s \_a m i n i @ y a h o o . c o m$ 


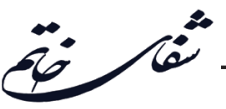

شناسايى هر پس ويروس انسانى تيب 6 و ويروس واريسلا -زوستر در ادرار بيماران مبتلا به مالتيّل اسكلروز در استان كرمان، ايروان

كيميا اسماعيلى، كيومرث امينى"

كروه ميكروبيولوزى، دانشكده علوم بايه، واحد ساوه، دانشكاه آزاد اسلامى، ساوه، ايران

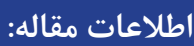

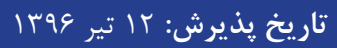

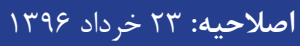

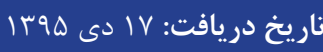

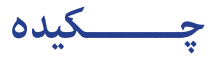

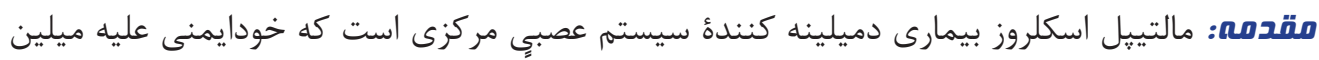

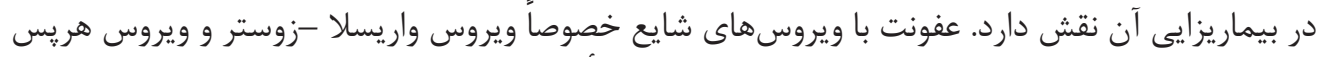

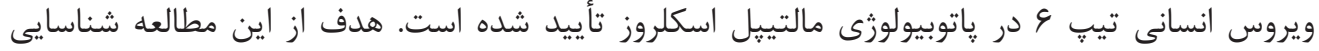

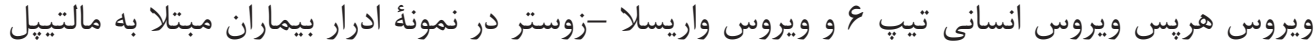

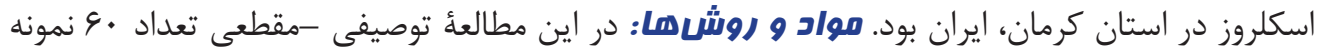

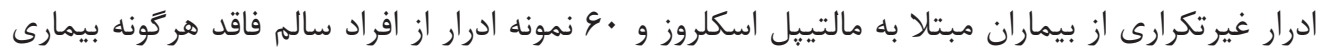

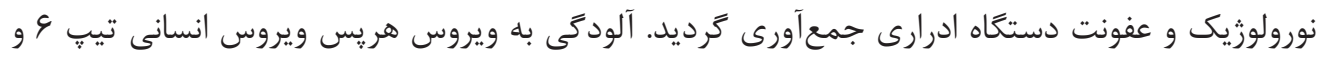

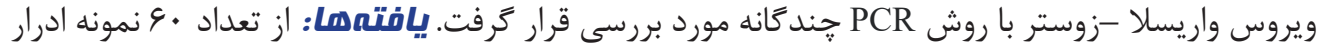

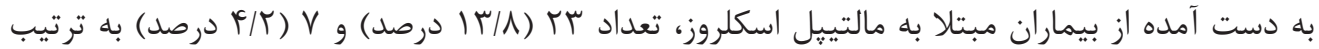

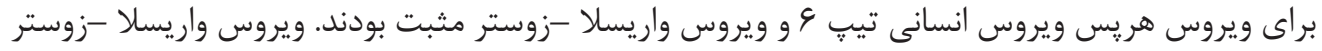

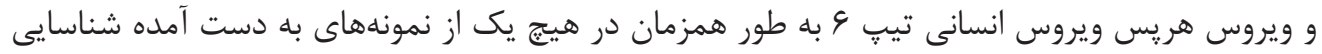

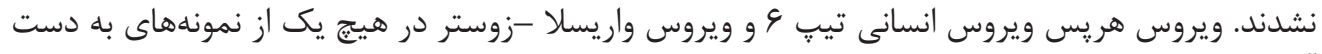

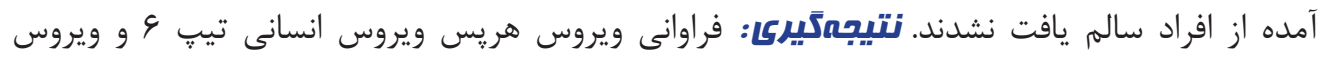

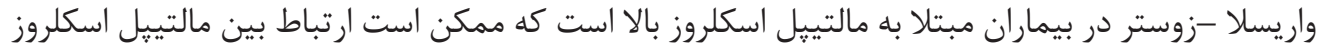

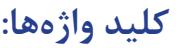
و حضور اين ويروسها در اين ناحيه را نشان دهد.

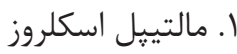

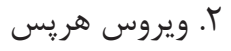

انسانى تيب

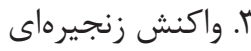

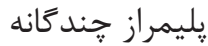

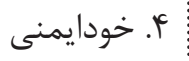

" نويسنده مسئول: كيومرث امينى

آدرس الكترونيكى: dr_kumarss_amini@yahoo.com 


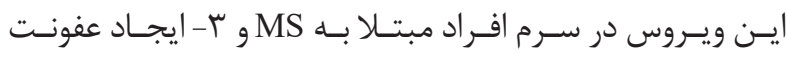

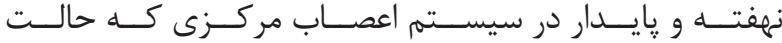

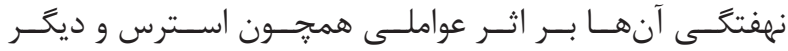

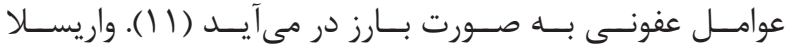

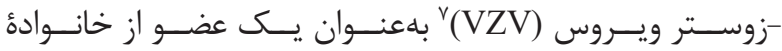

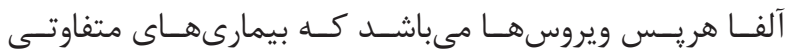

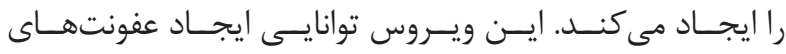

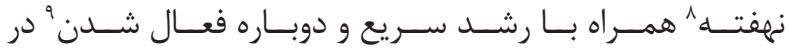

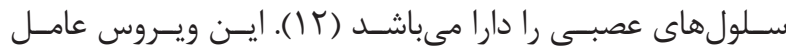

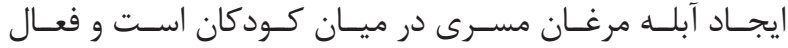

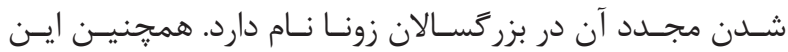

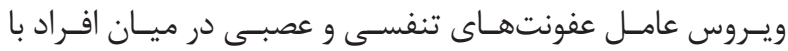

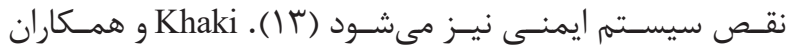

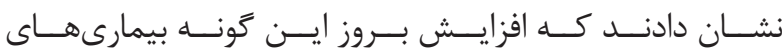

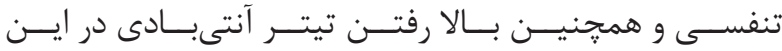

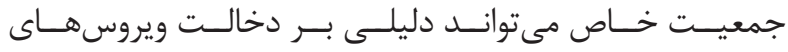

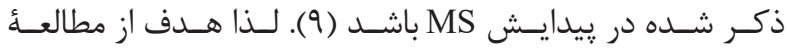

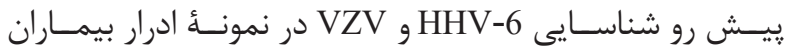

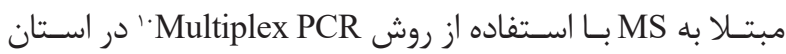

$$
\text { كرمــان مىباشـد. }
$$

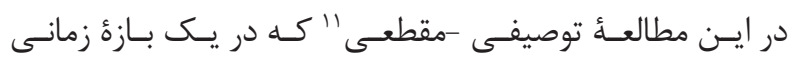

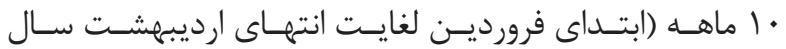

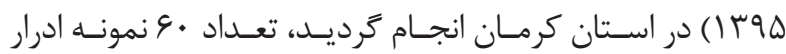

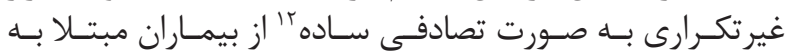

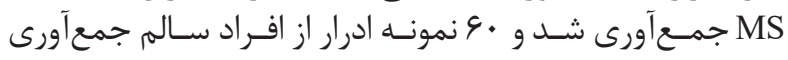

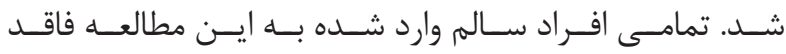

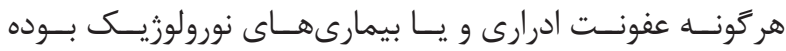

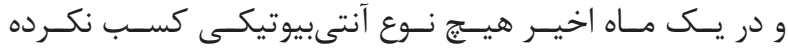

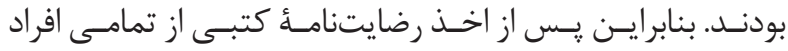

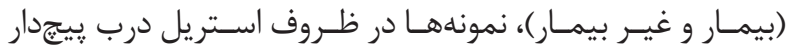

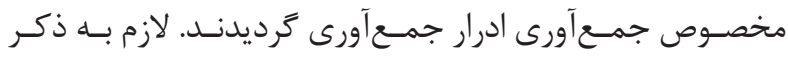

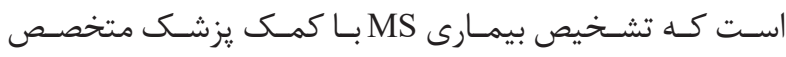

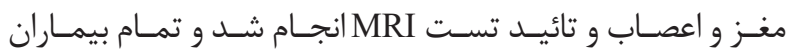

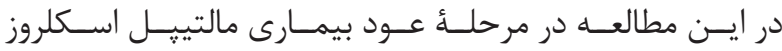
(MSRR)

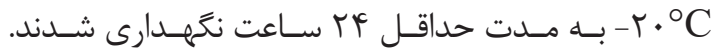

$$
\text { واكنش زنجيرهاى يليمراز جند }
$$

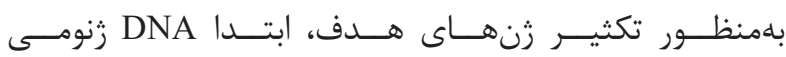

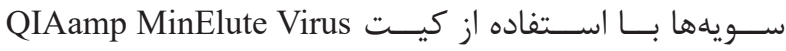

${ }^{1}$ Multiple sclerosis

${ }^{2}$ Relapsing remitting MS

${ }^{3}$ Primary progressive MS

${ }^{4}$ Secondary progressive MS

${ }^{5}$ Progressive relapsing MS

${ }^{6}$ Human herpesvirus 6

${ }^{7}$ Varicella zoster virus

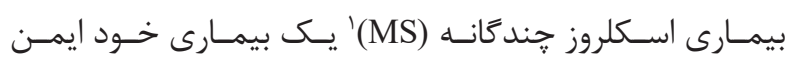

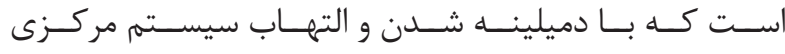

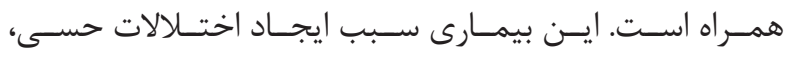

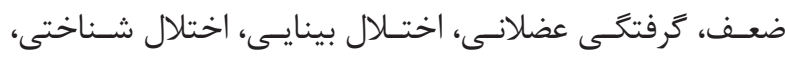

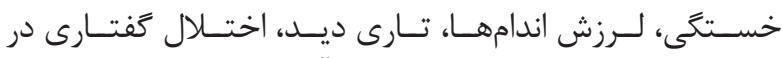

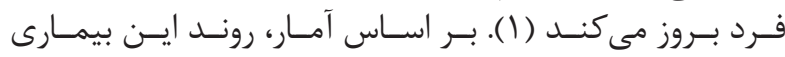

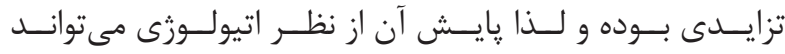

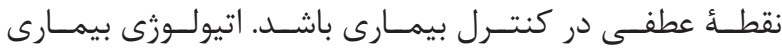

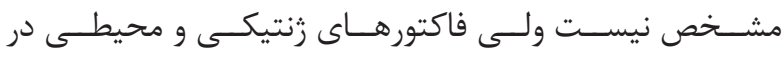

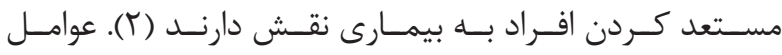

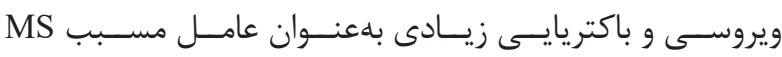

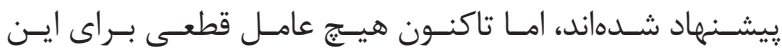

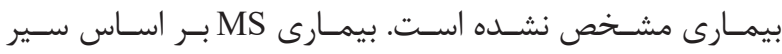

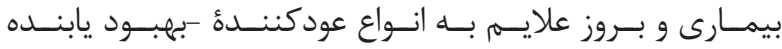
(RRMS)

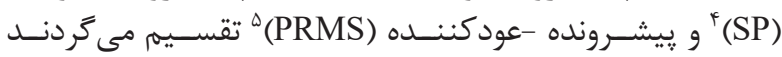

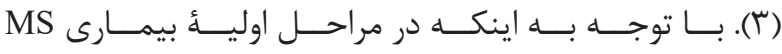

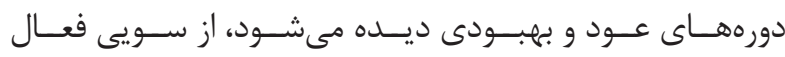

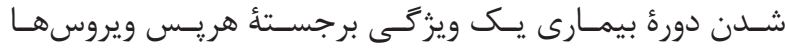

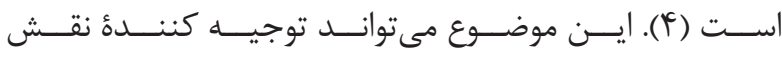

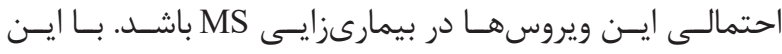

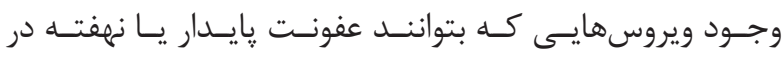

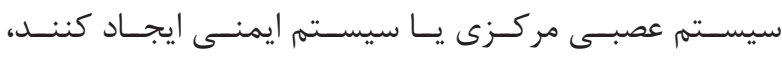

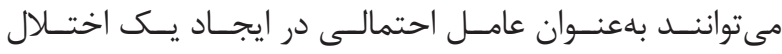

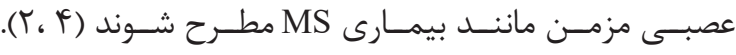
Taherkhani

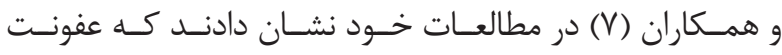

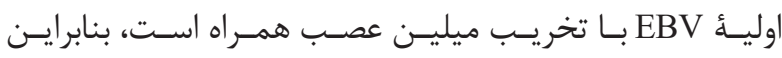

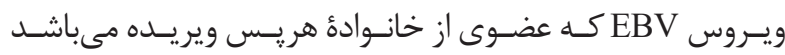

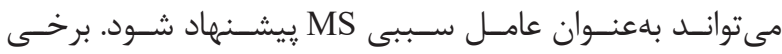

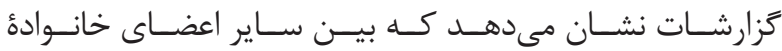

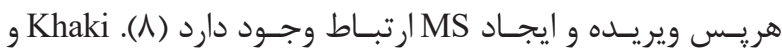

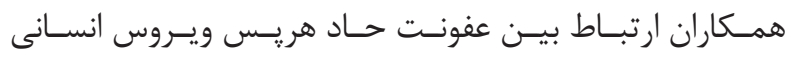

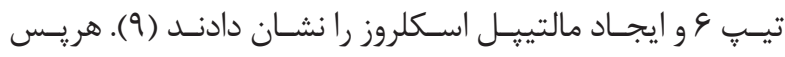
ويسروس انسـانى تيــ

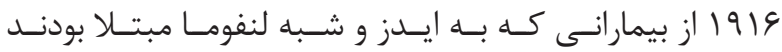

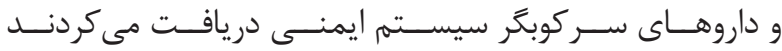

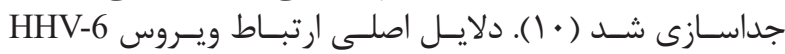

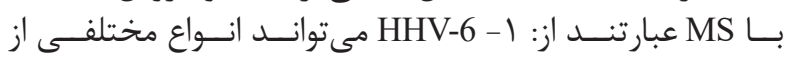

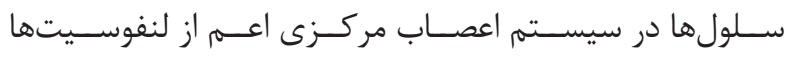

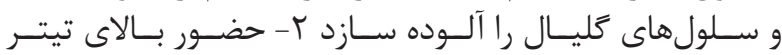

\footnotetext{
${ }^{8}$ Latent infection

${ }^{9}$ Reactivation

${ }^{10}$ Multiplex-polymerase chain reaction

${ }^{11}$ Cross-sectional study

${ }^{12}$ Simple random sampling

${ }^{13}$ Relapsing/remitting MS
} 


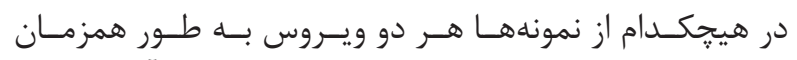

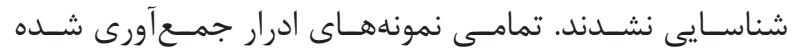

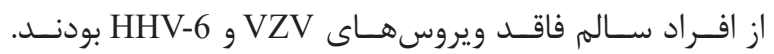

$$
\text { بحث و نتيجهَيرى }
$$

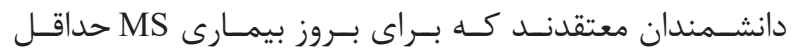

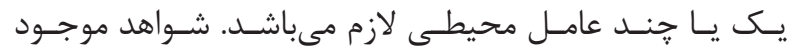

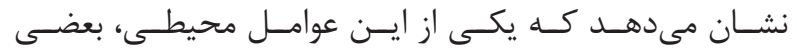

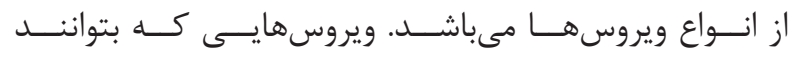

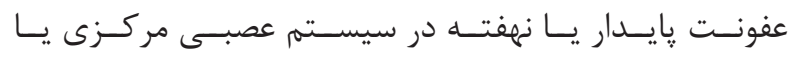

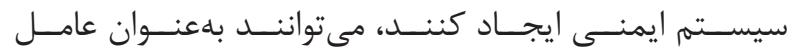

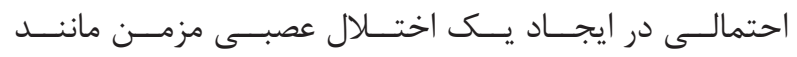

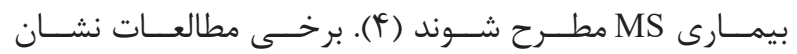

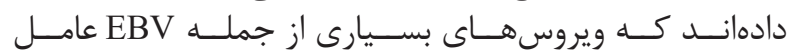

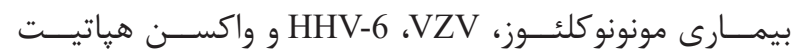

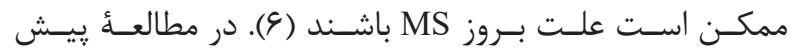

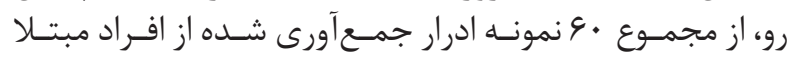

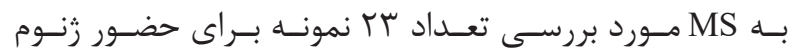

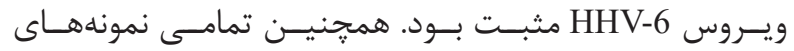

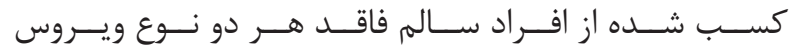

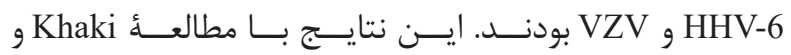

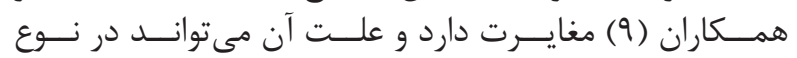

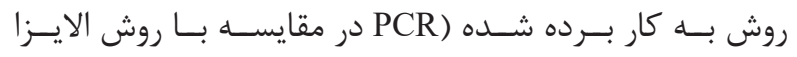

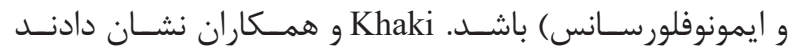

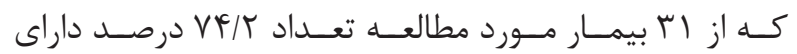

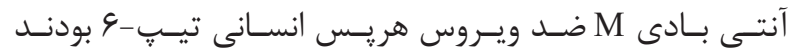

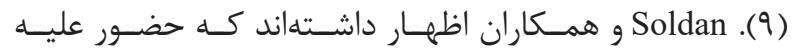

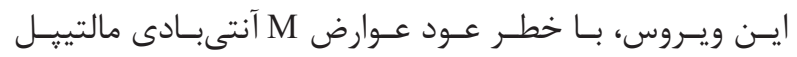

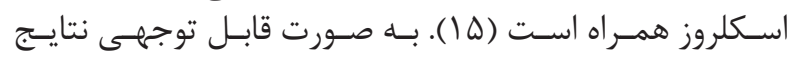

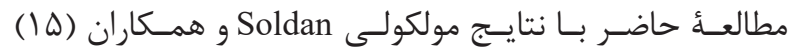

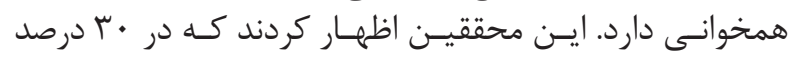

Spin Kit

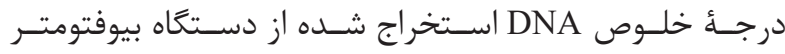

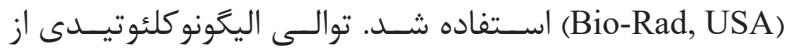

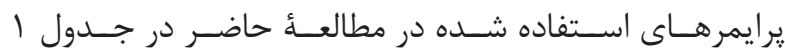

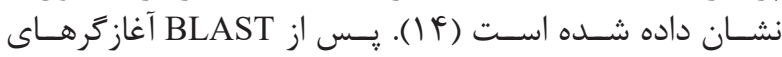

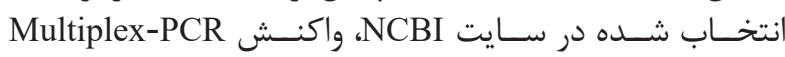
بـا master mix 5X $(\cdot / \uparrow \mathrm{mM}), \mathrm{MgCl} 2$ ( $\mathrm{FmM})$ (Taq DNA polymerase d dNTPs

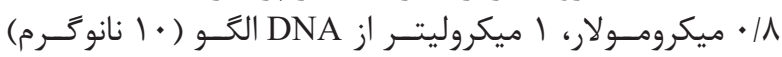

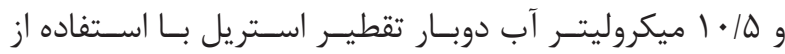

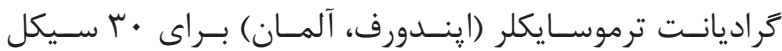

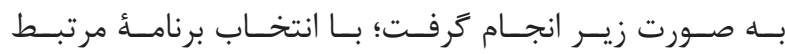

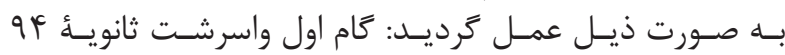

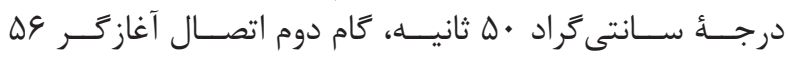

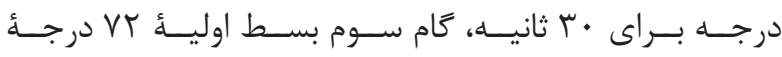

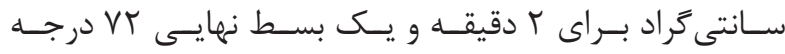

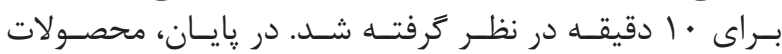

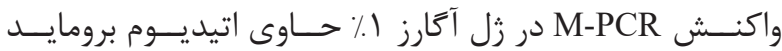

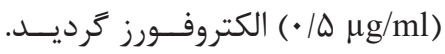

بافتهها

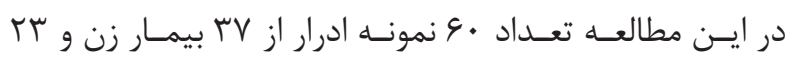

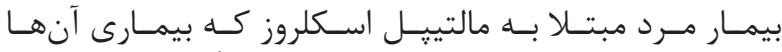

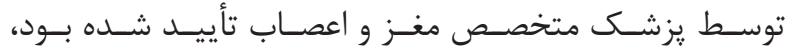

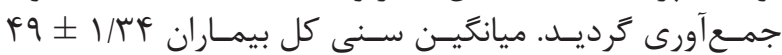

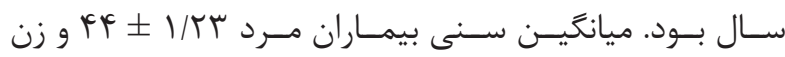

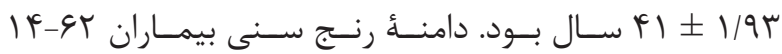

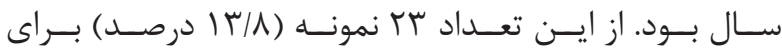

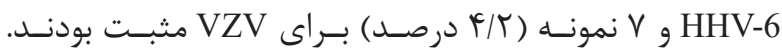

جدول ا- توالىهاى نوكلئوتيدى استفاده شده در اين تحقيق.

\begin{tabular}{|c|c|c|c|}
\hline ميكرواركانيسم & ل برايمرها & اندازه & منبع \\
\hline Human herpesvirus type 6 & $\begin{array}{l}\text { Forward: 5'-AAGCTTGCACAATGCCAAAAAACAG- } 3^{\prime} \\
\text { Reverse: 5'- CTCGAGTATGCCGAGACCCCTAATC-3' }\end{array}$ & far & (If) \\
\hline VZV & $\begin{array}{c}\text { Forward: 5'-ACGGGTCTTGCCGGAGCTGGT-3' } \\
\text { Reverse: 5'-AATGCCGTGACCACCAAGTATAAT-3' }\end{array}$ & $\Delta \Delta r$ & \\
\hline
\end{tabular}

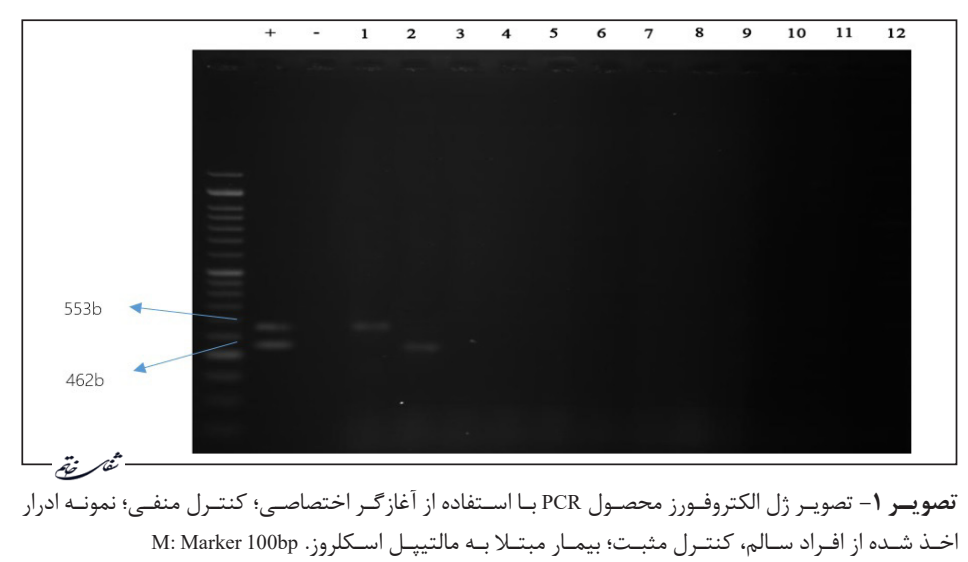




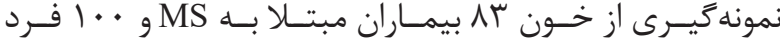

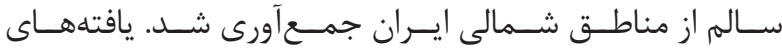

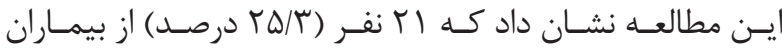

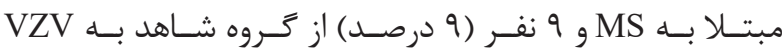

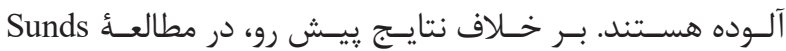

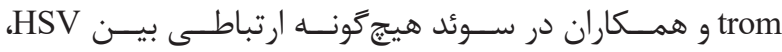

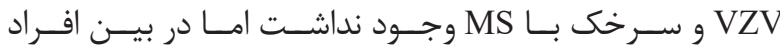

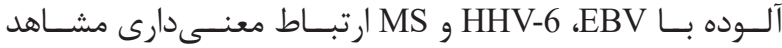

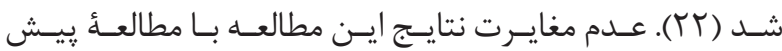

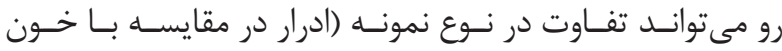

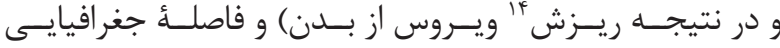

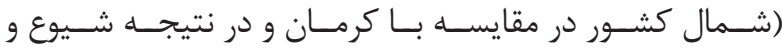

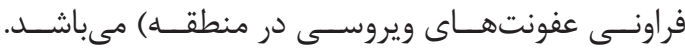

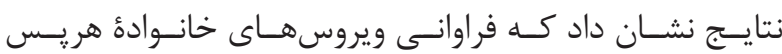

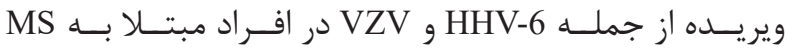

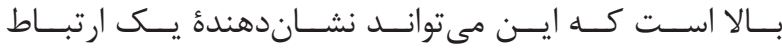

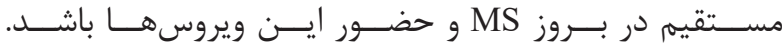

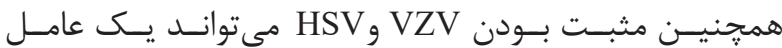

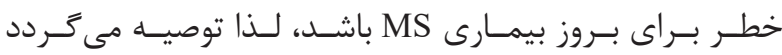

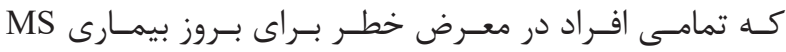

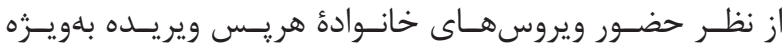

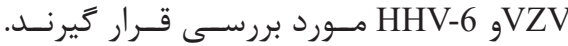

$$
\text { تشكر و قدردانى }
$$

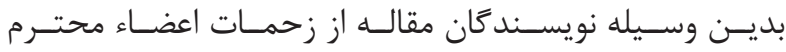

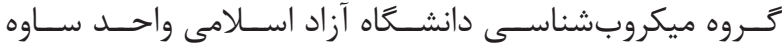

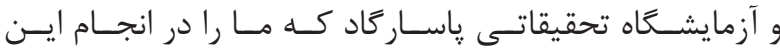

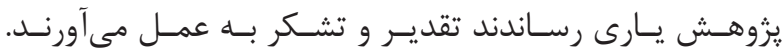

1. Paroni M, Maltese V, De Simone M, Ranzani V, Larghi P, Fenoglio C, et al. Recognition of viral and selfantigens by $\mathrm{T} \mathrm{H} 1$ and $\mathrm{T} \mathrm{H} \mathrm{1/T} \mathrm{H} 17$ central memory cells in patients with multiple sclerosis reveals distinct roles in immune surveillance and relapses. J Allergy Clin Immunol. 2017; 140(3): 797-808.

2. Martin R, Sospedra M, Rosito M, Engelhardt B. Current multiple sclerosis treatments have improved our understanding of MS autoimmune pathogenesis. Eur J Immunol. 2016; 46(9): 2078-90.

3. Cheng Y, Sun L, Xie Z, Fan X, Cao Q, Han J, et al. Diversity of immune cell types in multiple sclerosis and its animal model: Pathological and therapeutic implications. J Neurosci Res. 2017; 95(10): 1973-83.

4. Mentis A-FA, Dardiotis E, Grigoriadis N, Petinaki E, Hadjigeorgiou GM. Viruses and multiple sclerosis: from mechanisms and pathways to translational research

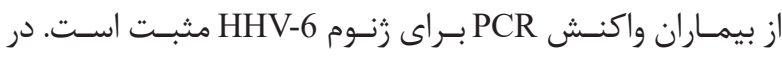

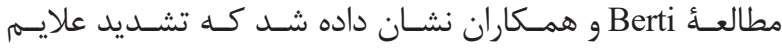

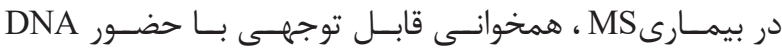

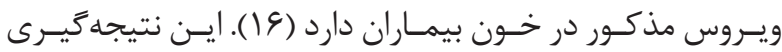

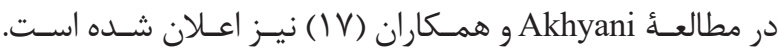
و همــار هawate

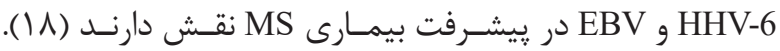

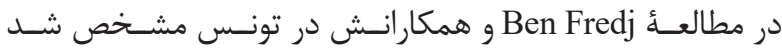

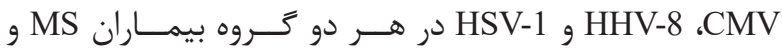

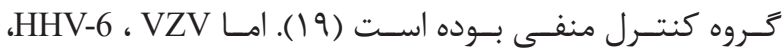

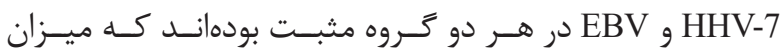

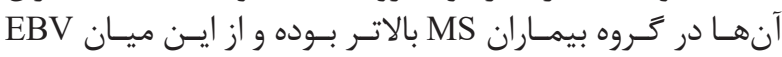

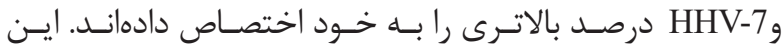

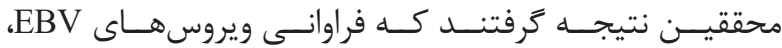

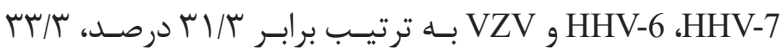

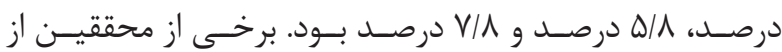

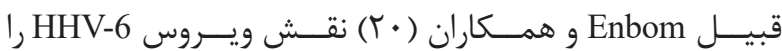

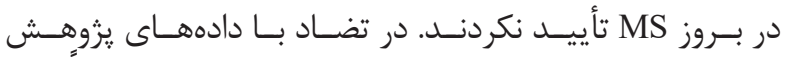

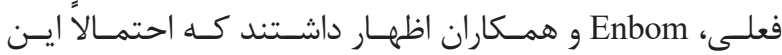

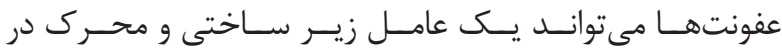

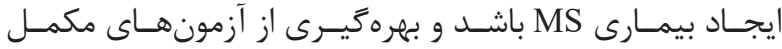

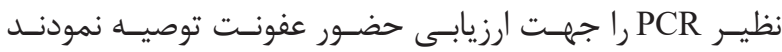

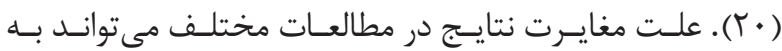

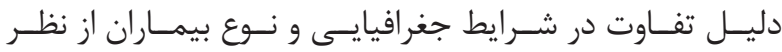

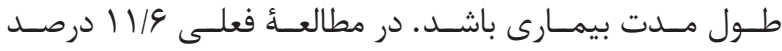

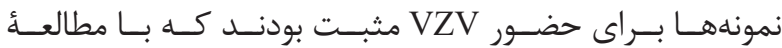
Najafi

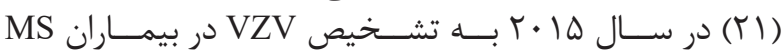

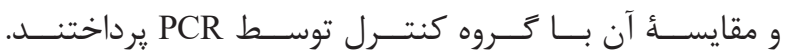

منابع

opportunities. Mol Neurobiol. 2017; 5(54): 3911-23.

5. Taherkhani R, Farshadpour F, Mirjalili A, Amirinejad R. Determination of the association between EpsteinBarr virus (EBV) infection and Multiple sclerosis (MS) disease. Iranian South Medical Journal. 2014; 17(4): 666-75.

6. Fujinami RS, von Herrath MG, Christen U, Whitton JL. Molecular mimicry, bystander activation, or viral persistence: infections and autoimmune disease. Clin Microbiol Rev. 2006; 19(1): 80-94.

7. Hon GM, Hassan MS, van Rensburg SJ, Erasmus RT, Matsha TE. Assessment of epstein-barr virus in blood from patients with multiple sclerosis. Metab Brain Dis. 2012; 27(3): 311-8.

8. Pormohammad A, Azimi T, Falah F, Faghihloo E. Relationship of human herpes virus 6 and multiple 


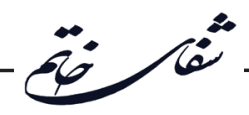

sclerosis: a systematic review and meta-analysis. J Cell Physiol. 2017. doi: 10.1002/jcp.26000.

9. Khaki M, Ghazaavi A, Ghasami K, Rafiei M, Payani M, Mosayebi G. Anti-human herpes virus-6 antibodies titer in patients with multiple sclerosis in Markazy Province. Arak Medical University Journal. 2009; 12(2): 45-50.

10. Levy JA. Three new human herpesviruses (HHV6, 7, and 8). Lancet. 1997; 349(9051): 558-63.

11. Ablashi D, Eastman H, Owen C, Roman M, Friedman J, Zabriskie J, et al. Frequent HHV-6 reactivation in multiple sclerosis (MS) and chronic fatigue syndrome (CFS) patients. J Clin Virol. 2000; 16(3): 179-91.

12. Mehta SK, Nelman-Gonzalez M, Tyring SK, Tong Y, Beitman A, Crucian BE, et al. Localization of VZV in saliva of zoster patients. J Med Virol. 2017; 89(9): 1686-9.

13. Karampoor S, Zahednasab H, Ramagopalan S, Mehrpour M, Etemadifar M, Alsahebfosoul F, et al. Cytomegalovirus and varicella zoster virus seropositivity of Iranian patients with multiple sclerosis: a populationbased study. J Neuroimmunol. 2017; 309: 4-6.

14. Gordon L, McQuaid S, Cosby S. Detection of herpes simplex virus (types 1 and 2) and human herpesvirus 6 DNA in human brain tissue by polymerase chain reaction. Clin Diagn Virol. 1996; 6(1): 33-40.

15. Soldan SS, Fogdell-Hahn A, Brennan MB, Mittleman BB, Ballerini C, Massacesi L, et al. Elevated serum and cerebrospinal fluid levels of soluble human herpesvirus type 6 cellular receptor, membrane cofactor protein in patients with multiple sclerosis. Ann Neurol. 2001; 50(4): 486-93.
16. Berti R, Brennan MB, Soldan SS, Ohayon JM, Casareto L, McFarland HF, et al. Increased detection of serum HHV-6 DNA sequences during Multiple Sclerosis (MS) exacerbations and correlation with parameters of MS disease progression. J Neurovirol. 2002; 8(3): 250-6.

17. Akhyani N, Berti R, Brennan MB, Soldan SS, Eaton JM, McFarland HF, et al. Tissue distribution and variant characterization of human herpesvirus (HHV)6: increased prevalence of HHV-6A in patients with multiple sclerosis. J Infect Dis. 2000; 182(5): 1321-5.

18. Pawate S, Sriram S. The role of infections in the pathogenesis and course of multiple sclerosis. Ann Indian Acad Neurol. 2010; 13(2): 80-6.

19. Ben Fredj N, Rotola A, Nefzi F, Chebel S, Rizzo R, Caselli E, et al. Identification of human herpesviruses 1 to 8 in Tunisian multiple sclerosis patients and healthy blood donors. J Neurovirol. 2012; 18(1): 12-9.

20. Enbom M, Wang FZ, Fredrikson S, Martin C, Dahl H, Linde A. Similar humoral and cellular immunological reactivities to human herpesvirus 6 in patients with multiple sclerosis and controls. Clin Diagn Lab Immunol. 1999; 6(4): 545-9.

21. Najafi S, Ghane M, Yousefzadeh-Chabok S, Amiri $M$. The high prevalence of the varicella zoster virus in patients with relapsing-remitting multiple sclerosis: a case-control study in the north of Iran. Jundishapur J Microbiol. 2016; 9(3). doi: 10.5812/jjm. 34158.

22. Sundström P, Juto P, Wadell G, Hallmans G, Svenningsson A, Nyström L, et al. An altered immune response to epstein-barr virus in multiple sclerosis a prospective study. Neurology. 2004; 62(12): 2277-82. 